\title{
A OVER DAMPED PERSON IDENTIFICATION SYSTEM USING EMG SIGNAL
}

\author{
Suresh $\mathbf{M}^{1}$, P G Krishnamohan ${ }^{2}$, Mallikarjun S Holi \\ ${ }^{1}$ Assistant Professor, Department of E\&C, KIT, Karnataka, India \\ ${ }^{2}$ Retired Professor, Department of E\&C, JNTUH, Hyderabad, India \\ ${ }^{3}$ Professor \& Chairman, Department of E\&I, UBDT, Karnataka, India
}

\begin{abstract}
EMG signal distinct from one person to another person. Body mass, muscle fiber pattern, muscle size, motion of subject, neuromuscular activity, neurotransmitter activity in different areas within the muscle, different density of bone, changes in blood flow in the muscle, fatigue, skin conductivity, motor unit firing pattern, motor unit paths, distribution of heat in the muscle, skinfat layer, motor unit recruitment order and characteristics of muscle, strength and force generated by the muscle changing from person to person. Change in measurement of EMG signal depends on many factors. Irrespective of which factor changes the EMG signal but physiologic dependent singularity is different and hence Electromyogram (EMG) can be used for person identification. Useful information present in EMG signal need to be extracted to discriminate different persons. In this work, EMG signal acquired from a single channel instrumentation system. Cepstrum technique used to extract features from EMG signal. The Vector Quantization (VQ) is used to build person models. 50 healthy persons EMG data is recorded in three sessions at different days. Person identification system developed using cepstrum and VQ provide good result in performance.
\end{abstract}

Keywords: Biometrics, cepstral features, feature extraction, vector quantization (VQ).

\section{INTRODUCTION}

Many parts of the body like fingers, hands, feet, ears, eyes, faces, teeth, veins characteristics of voice, signature, styles of typing, emotion, egos are used in biometric systems. Physiological signal like electrocardiogram(ECG) and electroencephalogram(EEG) can be used for person identification[1, 2, 3]. In 1970 komi and buskirk [4] found that the average test-retest reliability for surface electrodes was $88 \%$.

A train of impulses due to firing of motor units passes through the muscle gives motor unit action potential. The EMG signal can be modeled using

$$
Y(n)=i(r) f(n-r)+g(n)
$$

$\mathrm{F}(\mathrm{n})$ is impulse train, $\mathrm{i}(\mathrm{r})$ is impulse response, $\mathrm{g}(\mathrm{n})$ is Gaussian noise and $\mathrm{N}$ is number of motor units. Source is the impulse process, system is muscle, Cepstrum is used to separate sorce and system component and then person identification performed.

\section{EMG FEATURE EXTRACTION}

\section{Cepstral Features}

The time-frequency methods are used in EMG feature extraction because of short time variability of spectrum. The feature vectors extraction is done through cepstral coefficients. The cepstrum is obtained by taking the inverse Fourier transform of the $\log$ magnitude spectrum for an EMG signal.
Consider a given EMG burst for a particular person. EMG frames of size $50 \mathrm{~ms}$ (100 samples at $2 \mathrm{kHz}$ sampling rate) with a shift of $25 \mathrm{~ms}$ (50 samples at $2 \mathrm{kHz}$ sampling rate) are taken. Here assumption is the data within a window are nearly stationary. 40 frames per second and therefore 400 frames for every ten second slot of EMG data obtained. In each session five slots of ten seconds EMG data from all subjects has been collected. So for three sessions 6000 frames per subject are obtained. For each observation window, the first 13 basic cepstral coefficients plus 13 delta and 13 delta-delta coefficients were used as EMG signal features. Encouraging results obtained for 13 dimensional base cepstrum with 13 velocity and 13 acceleration coefficients.

\section{EMG MODELING USING VQ METHOD}

Person models built using the feature vectors from different persons EMG bursts. The desirable attributes of person model are better representation of person, must consume less time for processing and space complexity [6]. The objective of person identification is to obtain reliable performance. The success of modeling techniques depends on better cluster or captures the distribution of the feature vectors. Earlier studies for person identification used direct template matching between training and testing data $[7,8,9$, $10,11,12,13$,$] . It is common to reduce the number of$ feature vectors by clustering approach. The cluster centers are known as code vectors and a set of code vectors is known as codebook. Person identification system uses VQ modeling has all of these attributes, which is briefly described in this section. 


\section{Vector Quantization (VQ)}

After obtained cepstral feature vectors, second level of compression is done using VQ method, where a set of nearest feature vectors are clustered together. VQ model is formed for every person and comparison is done with the person VQ model. The binary split algorithm is used for obtaining codebook vectors from cepstral features, which in turn uses the K-means clustering algorithm [14, 15, 16]. Larger codebook and EMG test data required for getting good person identification performance. Also, VQ codebook can be updated to alleviate the performance degradation. The number of centroids should be at least ten times smaller than number of feature vectors in the EMG signal.

\section{RESULTS AND DISCUSSIONS}

Each session contains 5 slots of 10 seconds EMG data. There are total of 15 slots of data from all the three sessions. First four slots of any two arbitrary sessions are used for training. 400 feature vectors obtained for every 10 seconds slot of EMG data. Therefore for four slots of any two sessions data $1600 \times 2=3200$ feature vectors are used for training. The codebooks of all persons are stored in the EMG database. For testing, last slots (400 frames per slot) of all sessions are used separately.

Feature vector consist of 39 dimension coefficients including 13 dimensions of cepstral coefficients combined with its velocity and acceleration coefficients. The 39 dimension feature vectors are trained. The result of person identification performance for $\mathrm{VQ}$ technique is given in Table 1.

Table -1: 13 dimensional base cepstra plus, $13 \Delta$ and $13 \Delta \Delta$ with VQ

\begin{tabular}{|l|l|l|l|l|}
\hline Training & \multirow{2}{*}{$\begin{array}{l}\text { Code book } \\
\text { sessions }\end{array}$} & \multicolumn{3}{|l|}{ Testing session } \\
\cline { 3 - 5 } & $\mathbf{1 6}$ & 55.10 & 77.55 & 63.27 \\
\hline \multirow{3}{*}{$\mathbf{1}, \mathbf{1 6}$} & $\mathbf{3 2}$ & 59.18 & $\mathbf{8 3 . 6 7}$ & 61.23 \\
\cline { 2 - 5 } & $\mathbf{6 4}$ & 55.10 & 79.59 & 57.14 \\
\hline \multirow{3}{*}{$\mathbf{2 , 3}$} & $\mathbf{1 6}$ & 44.90 & 69.39 & $\mathbf{8 5 . 7 1}$ \\
\cline { 2 - 5 } & $\mathbf{3 2}$ & 46.94 & 69.39 & 83.67 \\
\cline { 2 - 5 } & $\mathbf{6 4}$ & 4490 & 69.39 & 81.63 \\
\hline \multirow{3}{*}{$\mathbf{1}, \mathbf{3}$} & $\mathbf{1 6}$ & 44.90 & 69.39 & $\mathbf{8 5 . 7 1}$ \\
\cline { 2 - 5 } & $\mathbf{3 2}$ & 46.90 & 71.43 & 83.67 \\
\cline { 2 - 5 } & $\mathbf{6 4}$ & 44.90 & 71.43 & 81.63 \\
\hline
\end{tabular}

During the course of experiment, 4 slots of EMG data from session 1 and session 2 are used for training and no slots of data from session 3 is used for training and remaining one slot of data from each of the session 1, session 2 and any of the slot of data of 10 seconds from session 3 are used for testing. Maximum person identification performance of $83.67 \%$ is obtained for the last slot of test EMG data chosen from trained session 2 for the 32 code book size rather than from session 1 test EMG data. It is obvious for the reason that the model is most recently learned with the session 2 data. It seems model is not yet generalized to yield better performance for the last slot of test EMG data from session 1and session 3. Further, the model is not trained with session 3 data and hence the maximum identification performance of $63.27 \%$ is obtained for the last slot of test EMG data selected from untrained session 3, which is less than the person identification performance of $83.67 \%$ for the last slot of test EMG data chosen from trained session 2.

This model is similarly trained with session 2 and session 3 EMG data without using session 1 EMG data for training. In this case, maximum person identification performance of $85.71 \%$ is obtained for the last slot of test EMG data selected from trained session 3 with code book size of 16 . Since the session 1 EMG data is not used for training the model, the maximum person identification performance of $46.94 \%$ is obtained for the last slot of test EMG data selected from untrained session 1 for the codebook size of 32 which is less than the maximum person identification performance of $85.71 \%$ obtained for the last slot of test EMG data selected from trained session 3 with code book size of 16 . In this case also, the model yield better performance $85.71 \%$ for the last slot of 10 seconds of EMG data selected from the most recently learned session 3 EMG data.

In order to complete the experiment, the model is again trained with session 1 and session 3 EMG data without using the session 2 EMG data for training. In this case, the model yields the maximum person identification performance of $85.71 \%$ with codebook size of 16 for the last slot of 10 seconds of test EMG data selected from trained session 3 which is also the most recently learned session EMG data by the model. Further, the model yields maximum person identification performance of $71.43 \%$ with code book size of 32 and 64 for last slot of 10 seconds of EMG data selected from untrained session 2. This result is again less than the result obtained from the trained session 3 .

It is evident from these set of experiments that the model is suffering from session generalization and is varying from experiment to experiment for the test EMG data selected from untrained sessions. In order to confirm this findings, the experiment is again repeated with sessions used for training is reversed and similar observation is found in Table 2.

Table -2: Cepstra-VQ with two sessions used for training is reversed

\begin{tabular}{|l|l|l|l|l|}
\hline Training & \multirow{2}{*}{$\begin{array}{l}\text { Code } \\
\text { sessions } \\
\text { book } \\
\text { beversed }\end{array}$} & \multicolumn{4}{|l|}{ Testing session } \\
\cline { 2 - 5 } & size & $\mathbf{1}$ & $\mathbf{2}$ & $\mathbf{3}$ \\
\hline \multirow{3}{*}{$\mathbf{2 , 1}$} & $\mathbf{1 6}$ & 81.63 & 38.77 & 42.86 \\
\cline { 2 - 5 } & $\mathbf{3 2}$ & 85.71 & 40.82 & 34.69 \\
\cline { 2 - 5 } & $\mathbf{6 4}$ & $\mathbf{8 7 . 7 5}$ & 32.65 & 38.78 \\
\hline \multirow{3}{*}{3,2} & $\mathbf{1 6}$ & 55.10 & 77.55 & 61.23 \\
\cline { 2 - 5 } & $\mathbf{3 2}$ & 55.10 & $\mathbf{8 1 . 6 3}$ & 61.23 \\
\cline { 2 - 5 } & $\mathbf{6 4}$ & 57.14 & 77.55 & 57.14 \\
\hline \multirow{3}{*}{$\mathbf{3 , 1}$} & $\mathbf{1 6}$ & 81.63 & 38.78 & 44.90 \\
\cline { 2 - 5 } & $\mathbf{3 2}$ & 83.67 & 40.82 & 34.69 \\
\cline { 2 - 5 } & $\mathbf{6 4}$ & $\mathbf{8 5 . 7 1}$ & 30.61 & 36.74 \\
\hline
\end{tabular}


(a) 1,2

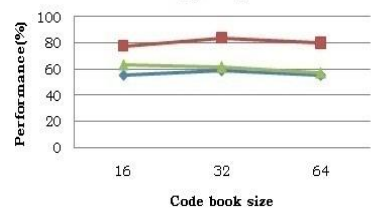

(b) 2,3

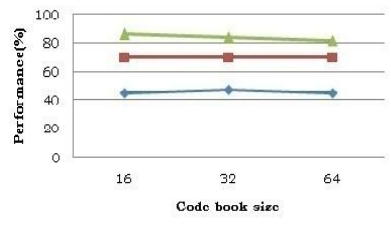

(c) 1,3

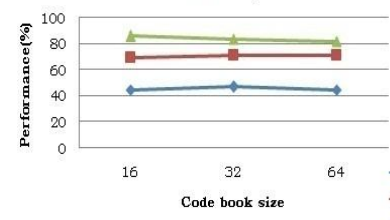

Fig -1: Performance of person identification system based on 13 dimensional base cepstra plus, $13 \Delta$ and13 $\Delta \Delta$ with VQ

The Fig. 1, shows the large person identification performance for recently trained sessions. From results, it is well understood that the problem is in session generalization. Further to explore, the EMG data slots are intermixed amongst the sessions selected for training and above experiment is repeated with an expectation of model person identification performance improvements. For this purpose, following mixing strategy is employed. In the proposed mixing strategy, first slot of 10 seconds of EMG data of session 1 and session 2 are first used for training the model and then the second slot of 10 seconds of EMG data of both sessions are similarly used next for training the model and so on. In this case, after similar testing, maximum person identification performance of $83.67 \%$ and $71.43 \%$ is obtained for last slot of 10 seconds of test EMG data selected from session 1 and session 2 respectively with improved session generalization amongst training session as shown in Table 3. In this case also, the model suffers from session generalization for test EMG data selected from untrained session as shown in Fig.2.

Table -3: Cepstra-VQ with two sessions used for training is intermixed

\begin{tabular}{|l|l|l|l|l|}
\hline Training $\begin{array}{l}\text { Tessions with } \\
\text { session } \\
\text { intermixed } \\
\text { data slots }\end{array}$ & $\begin{array}{l}\text { Code } \\
\text { book } \\
\text { size }\end{array}$ & $\mathbf{1}$ & $\mathbf{2}$ & \multirow{2}{|l|}{ Testing session } \\
\cline { 2 - 5 } & $\mathbf{1 6}$ & $\mathbf{8 3 . 6 7}$ & 71.43 & 55.10 \\
\hline \multirow{3}{*}{$\mathbf{1 , 2}$} & $\mathbf{3 2}$ & 79.59 & $\mathbf{7 1 . 4 3}$ & 46.94 \\
\cline { 2 - 5 } & $\mathbf{6 4}$ & 77.55 & 69.40 & 48.98 \\
\hline \multirow{3}{*}{$\mathbf{2 , 3}$} & $\mathbf{1 6}$ & 51.02 & 85.71 & $\mathbf{7 1 . 4 3}$ \\
\cline { 2 - 5 } & $\mathbf{3 2}$ & 53.10 & $\mathbf{8 7 . 7 6}$ & 69.40 \\
\hline \multirow{3}{*}{$\mathbf{1 , 3}$} & $\mathbf{6 4}$ & 53.10 & 85.71 & 71.43 \\
\hline & $\mathbf{1 6}$ & 69.39 & 67.40 & 63.27 \\
\cline { 2 - 5 } & $\mathbf{3 2}$ & $\mathbf{7 7 . 6 0}$ & 65.31 & $\mathbf{7 1 . 4 3}$ \\
\cline { 2 - 5 } & $\mathbf{6 4}$ & 83.67 & 55.10 & 65.31 \\
\hline
\end{tabular}

1,2

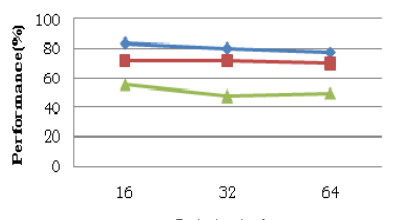

Code book size

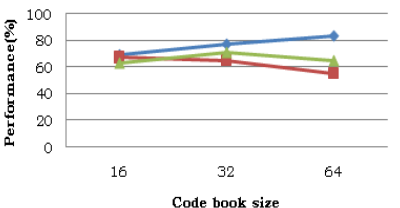

Code book size
2,3

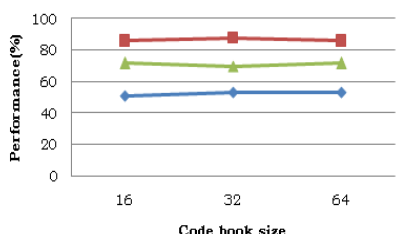

Code book size

$\rightarrow$ Session1

- - - Session2

- Session 3
Fig -2: Performance of person identification system with two sessions used for training is intermixed using CepstraVQ

This problem can be overcome by choosing all the three sessions of EMG data for training. In order to explore this fact, intermixing of slots of 10 seconds of data from all the three sessions is employed for training. In a conventional manner the last slot of 10 seconds of test EMG data from each of the trained session is used for testing. In this circumstance, the model yield maximum person identification performance of $83.67 \%$ for a codebook size of 16 and 32 for session 1 and session 2 respectively and $65.31 \%$ for codebook size of 16 for session 3 as shown in Table 4. Further, the same consistent person identification performance is observed when sessions are interchanged during training with testing procedure is repeated. It is observed that session generalization amongst trained sessions is improved with enhanced performance shown in Fig.3.

Table -4: Cepstra-VQ with three sessions for training intermixed

\begin{tabular}{|l|l|l|l|l|}
\hline \multirow{2}{*}{$\begin{array}{l}\text { Training } \\
\text { sessions }\end{array}$} & $\begin{array}{l}\text { Code } \\
\text { book } \\
\text { size }\end{array}$ & \multicolumn{4}{|l|}{ Testing session } \\
\cline { 2 - 5 } & $\mathbf{1}$ & $\mathbf{2}$ & $\mathbf{3}$ \\
\hline \multirow{4}{*}{$\mathbf{1 , 2 , 3}$} & $\mathbf{1 6}$ & $\mathbf{8 3 . 6 7}$ & 81.63 & $\mathbf{6 5 . 3 1}$ \\
\cline { 2 - 5 } & $\mathbf{3 2}$ & 79.59 & $\mathbf{8 3 . 6 7}$ & 63.27 \\
\cline { 2 - 5 } & $\mathbf{6 4}$ & 73.47 & 79.59 & 63.27 \\
\hline \multirow{3}{*}{$\mathbf{2 , 3 , 1}$} & $\mathbf{1 6}$ & $\mathbf{8 3 . 6 7}$ & 81.63 & $\mathbf{6 5 . 3 1}$ \\
\cline { 2 - 5 } & $\mathbf{3 2}$ & 79.59 & $\mathbf{8 3 . 6 7}$ & 63.27 \\
\cline { 2 - 5 } & $\mathbf{6 4}$ & 73.47 & 79.59 & 63.27 \\
\hline \multirow{3}{3}{$\mathbf{3 , 2}$} & $\mathbf{1 6}$ & $\mathbf{8 3 . 6 7}$ & 81.63 & $\mathbf{6 5 . 3 1}$ \\
\cline { 2 - 5 } & $\mathbf{3 2}$ & 79.59 & $\mathbf{8 3 . 6 7}$ & 63.27 \\
\cline { 2 - 5 } & $\mathbf{6 4}$ & 73.47 & 79.59 & 63.27 \\
\hline
\end{tabular}




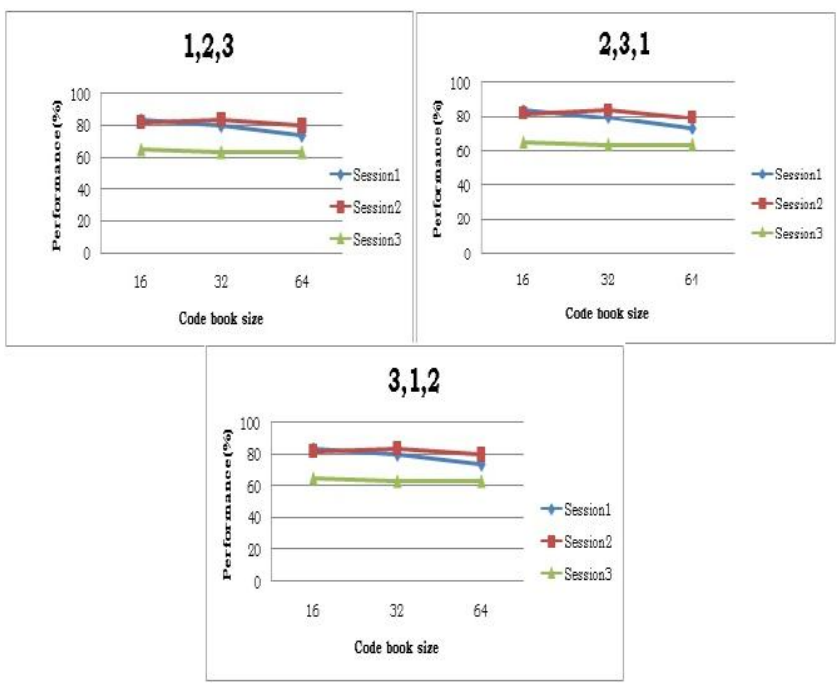

Table 5 describes the person identification performance for first four slots of any arbitrary chosen two sessions for training using $\mathrm{VQ}$ and all the individual five slots of remaining (untrained) session for testing separately to verify the consistency of result for the entire slots. Promising person identification performance has obtained for all the slots of untrained session.

Fig -3: Performance of person identification system with three sessions used for training is intermixed using Cepstra-

VQ

Table -5: Results for all individual slots of untrained session

\begin{tabular}{|c|c|c|c|c|c|c|}
\hline \multirow[t]{2}{*}{ Training sessions } & \multirow{2}{*}{$\begin{array}{l}\text { Code book } \\
\text { size }\end{array}$} & \multicolumn{5}{|c|}{ Testing } \\
\hline & & Slot1 & Slot2 & Slot3 & Slot4 & Slot5 \\
\hline \multirow[t]{3}{*}{1,2} & \multirow{3}{*}{$\begin{array}{l}16 \\
32 \\
64\end{array}$} & 79.59 & 73.47 & 71.43 & 71.44 & 71.43 \\
\hline & & 73.47 & 73.47 & 75.51 & 69.39 & 69.39 \\
\hline & & 79.59 & 75.51 & 73.47 & 69.39 & 65.31 \\
\hline \multirow[t]{3}{*}{2,3} & \multirow{3}{*}{$\begin{array}{l}16 \\
32 \\
64\end{array}$} & 53.06 & 46.94 & 57.14 & 57.14 & 57.14 \\
\hline & & 53.06 & 48.98 & 59.18 & 61.22 & 53.06 \\
\hline & & 46.94 & 51.02 & 53.06 & 57.14 & 51.02 \\
\hline \multirow[t]{3}{*}{1,3} & \multirow{3}{*}{$\begin{array}{l}16 \\
32 \\
64\end{array}$} & 61.22 & 79.59 & 71.43 & 77.55 & 77.53 \\
\hline & & 63.26 & 73.47 & 67.35 & 77.55 & 71.42 \\
\hline & & 67.35 & 77.55 & 6533 & 73.46 & 71.42 \\
\hline
\end{tabular}

The Fig.4. Shows performance of $65 \%$ to $79 \%$ with session 1, 2 trained and session 3 all slots used for testing separately. $47 \%-61 \%$ performance when session 2, 3 trained and session 1 all slots used for testing individually and 61\%$79 \%$ when session 1, 3 trained and remaining five slots of session 2 used for testing separately. For all code book size of VQ and slots of untrained session person identification performance is almost similar.
1,2

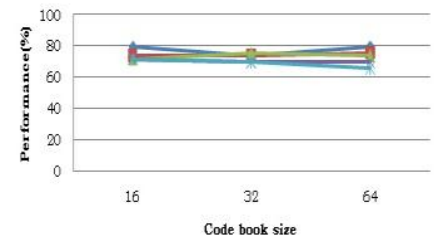

1,3

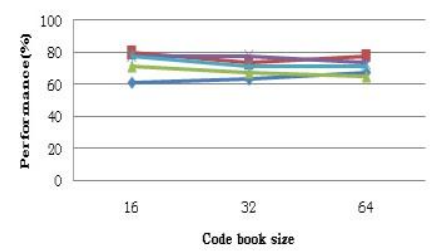

Fig -4: Performance of person identification system for all individual slots of untrained session with cepstra-VQ

To customize the EMG based person identification system the experiment is repeated for different group of persons and tested for up to 1024 sized code books and the results are shown in Table 6. 
Table -6: cepstra-VQ with three sessions used for training intermixed for different groups of people

\begin{tabular}{|l|l|l|l|l|l|l|l|l|l|l|}
\hline \multirow{2}{*}{$\begin{array}{l}\text { Total } \\
\text { No. of persons }\end{array}$} & \multicolumn{9}{l|}{ Code book size } \\
\cline { 2 - 11 } & $\mathbf{2}$ & $\mathbf{4}$ & $\mathbf{8}$ & $\mathbf{1 6}$ & $\mathbf{3 2}$ & $\mathbf{6 4}$ & $\mathbf{1 2 8}$ & $\mathbf{2 5 6}$ & $\mathbf{5 1 2}$ & $\mathbf{1 0 2 4}$ \\
\hline $\mathbf{0 5}$ & 100 & 100 & 100 & 100 & 100 & 100 & 100 & 100 & 100 & 80 \\
\hline $\mathbf{1 0}$ & 70 & 60 & 80 & 90 & 70 & 50 & 80 & 90 & 80 & 70 \\
\hline $\mathbf{1 5}$ & 53 & 33 & 60 & 66 & 53 & 40 & 60 & 66 & 60 & 53 \\
\hline $\mathbf{2 0}$ & 50 & 30 & 65 & 75 & 55 & 45 & 65 & 65 & 55 & 50 \\
\hline $\mathbf{2 5}$ & 56 & 40 & 68 & 76 & 64 & 52 & 68 & 68 & 60 & 56 \\
\hline $\mathbf{3 0}$ & 60 & 43 & 70 & 76 & 66 & 56 & 70 & 70 & 63 & 60 \\
\hline $\mathbf{3 5}$ & 62 & 51 & 62 & 80 & 71 & 62 & 74 & 71 & 68 & 65 \\
\hline $\mathbf{4 0}$ & 62 & 57 & 67 & 80 & 75 & 67 & 77 & 75 & 70 & 70 \\
\hline $\mathbf{4 5}$ & 64 & 62 & 71 & 82 & 77 & 71 & 80 & 77 & 73 & 73 \\
\hline $\mathbf{5 0}$ & 67 & 65 & 73 & 83 & 79 & 73 & 81 & 77 & 69 & 73 \\
\hline \hline
\end{tabular}

The variation in performance of EMG person identification system for different codebook size is graphically illustrated in Fig. 5. It is evident from the graph that the performance gradually increases from code book size of 2 and become consistent from code book size of 64 onwards. The nature of variation of performance with changing code book size resembles mostly the behavior of over damped system. The performance of EMG based person identification system become substantially stable from code book size of 64 onwards for change in total number of persons. In order to ensure this fact, the performance change against the variation in total number of persons is graphically illustrated in Fig. 6, given below. It is clearly revealed the fact that the performance of this EMG based person identification system substantially remains stable from codebook size of 64 onwards for change in total number of persons. Thus the stability of EMG based person identification system that is developed in this work demonstrated excellent in its performance from code book size of 64 onwards and is superior at the codebook size of 128 .

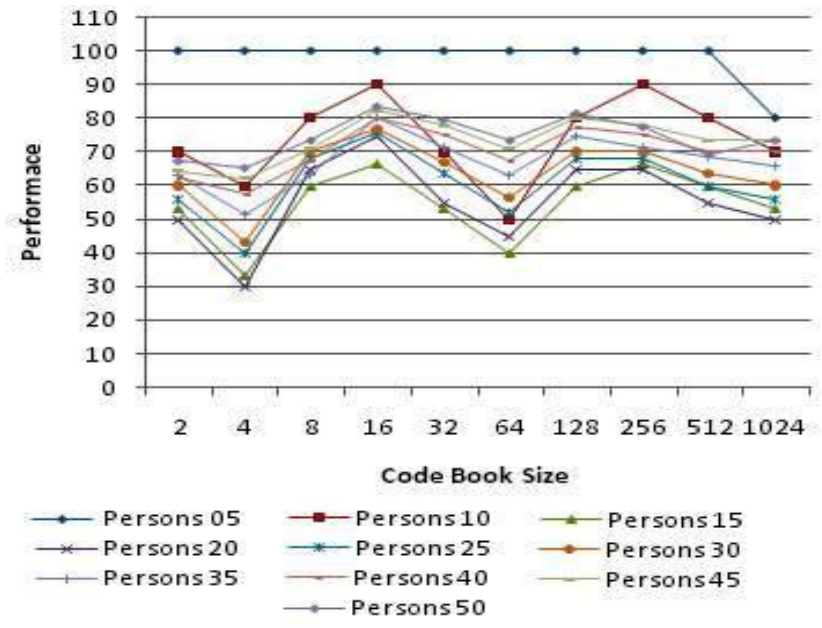

Fig -5: Performance variations versus code book size for intermixed 3 session data using cepstra-VQ.

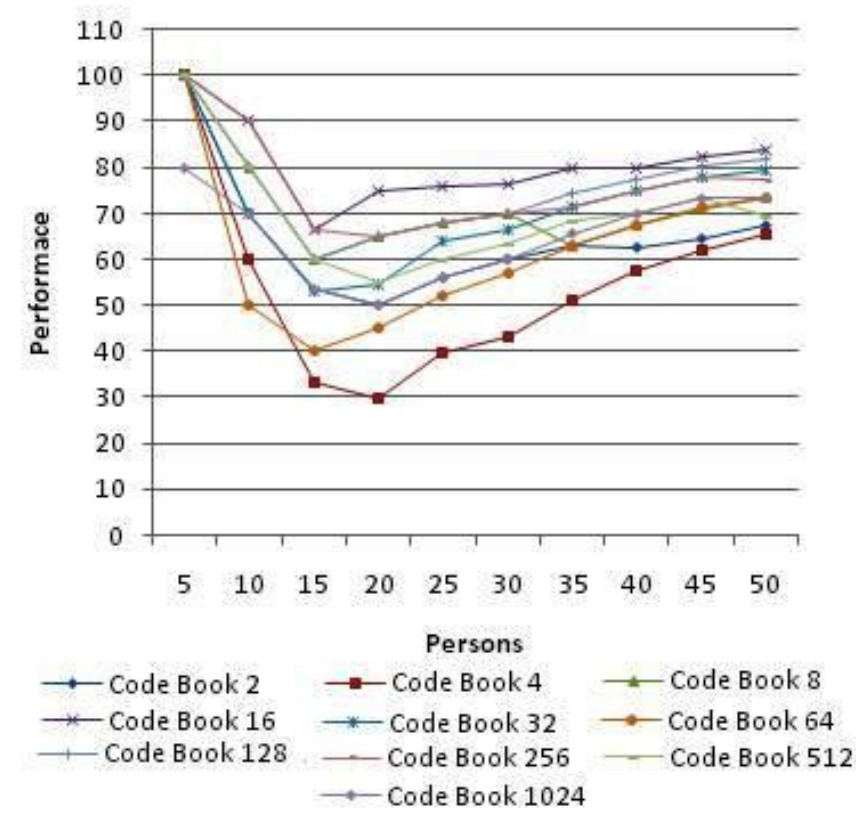

Fig -6: Performance variations versus group of persons for intermixed 3 session data using Cepstra-VQ.

\section{CONCLUSION}

An EMG based person identification system using 39 dimensional Cepstra-VQ approaches substantially remains stable from codebook size of 64 onwards for change in total number of persons. Thus the stability of EMG based person identification system that is developed in this work demonstrated excellent in its performance from code book size of 64 onwards and is superior at the codebook size of 128.

\section{REFERENCES}

[1] M. Poulos, et al," Neural Network based Person Identification using EEG Features," IEEE International Conference on Acoustics, Speech, and Signal Processing, volume 2: 1999, PP.1117-1120

[2] Israel. S, Irvine. J, Cheng. A, Widerhold. M, Widerhold. B,'ECG to identify individuals", Pattern Recognition 38(1), 2005, PP.133-142. 
[3] L. Biel, O. Pettersson, L. Philipson, and P. Wide. "ECG Analysis: A New Approach in Human Identification", IEEE Transactions on Instrumentation and Measurement, vol. 50, no. 3, June 2001, pp. $808-812$.

[4] Komi.P, Buskirk.E," Reproducibility of electromyographic measurements with inserted wire electrodes and surface electrodes," Electromyography 1970, 10:357.

[5] Suresh.M, P.G.Krishnamohan, Mallikarjun S Holi,” Significance of EMG in Biometric Person Authentication," In: the Proceedings of International Conference on Recent Innovations in Technology (ICRIT 2011), Feb 10-12, 2011, Kottayam, pp. 113118.

[6] D.A.Reynolds, “An overview of automatic speaker recognition technology," in Proc. IEEE Int. Conf. Acoust., Speech Signal Process, vol. 4, May 2002, pp. 4072-4075.

[7] James E. Luck, "Automatic speaker verification using cepstral measurements," J. Acoust. Soc. Amer., vol. 46(2), Nov. 1969, pp. 1026-1032.

[8] S.Pruzansky, "Pattern-matching procedure for automatic talker recognition," J. Acoust. Soc. Amer., vol. 35(3), March. 1963, pp. 354-358.

[9] J.W.Glenn and N.Kleiner, "Speaker identification based on nasal phonation ," J. Acoust. Soc. Amer., vol. 43(2), June 1967, pp. 368-372.

[10] B.S.Atal, "Effectiveness of linear prediction characteristics of the speech wave for automatic speaker identification and verification," J. Acoust. Soc. Amer., vol. 55, 1974, pp. 1304-1312.

[11] M.R.Sambur,"Selection of acoustic features for speaker identification," IEEE Trans. Acoust., Speech, Signal Process., vol. ASSP-23(2), Apr. 1975, pp. 176-182.

[12] A.E.Rosenberg and M.R.Sambur, "New techniques for automatic speaker verification," IEEE Trans. Acoust., Speech, Signal Process., vol. ASSP-23(2), Apr. 1975, pp. 169-176.

[13] M.R.Sambur,"Speaker recognition using orthogonal linear prediction," IEEE Trans. Acoust., Speech, Signal Process.., vol. ASSP-24(4), Aug. 1976, pp. 283-289.

[14] P.K. Soong, A.E. Rosenberg, L.R. Rabiner, B.H. Juang "A vector quantization approach to speaker recognition," AT\&T Tech. J., vol. 66, no. 2, 1987, pp. 14-26.

[15] Higgins, L.Bhaler, and J.Porter, "Voice identification using nearest neighbor distance measure," in Proc. IEEE Int. Conf. Acoustics, Speech, and Signal Processing, Minneapolis, MN, 1993, pp. 375-378.

[16] Y.Linde, A.Buzo, and R.M.Gray, "An algorithm for vector quantizer design," IEEE Trans. Communications, vol. COM-28(1), pp. 84-96, Jan. 1980.

[17] P.Dempster, N.M.Laird, and D.B. Rubin, "Maximum Likelihood from Incomplete Data via the EM algorithm," J. Roy. Statist. Soc. B, vol. 39, 1977, pp. 1-38.
[18] E.W.Theado, G.G.Knapik and W.S.Marras, "Modification of an EMG -assisted biomechanical model for pulling and pushing," in International Journal of Industrial Ergonomics, 37(2007)825-831.

\section{BIOGRAPHIES}

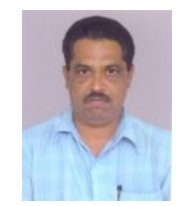

M.Suresh was born in India on October 28, 1967.He received the $\mathrm{BE}$ degree in instrumentation at Siddaganga Institute of Technology, Tumkur, Karnataka and ME degree in electronics from the BMS College of engineering, Bangalore, India in 1991 and 1994 respectively. From 1994 to 1999, he was a Lecturer, onwards and currently he is Assistant Professor with electronics and communication department, Kalpataru Institute of Technology, Tiptur, India, pursuing $\mathrm{PhD}$ programme at Jawaharlal Nehru Technological University, Kukatpally, Hyderabad, Andhrapradesh. His research interests include biometrics, biomedical and speech signal processing. Prof. Suresh is a member of ISTE and member of IETE.

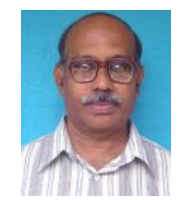

Dr.P.G.Krishnamohan was born in India on June 25, 1952. He received the $\mathrm{B}$. Tech degree in electronics and communication engineering at Regional Engineering College, Warangal in 1975. He received the master degree in Communication Systems at Rookee University, Roorkee, Uttar Pradesh, India in 1977 and research degree in Signal Processing at Indian Institute of Science, Bangalore, India in 1988. He has 36 years teaching experience, Worked as Head of Department of Electronics and Communication Engineering Department, JNTU, Hyderabad. he guided 30 M.Tech student project and $3 \mathrm{PhD}$ students. Many research projects carried out successfully and published 42 research papers. He was chairman of board of studies of E.C.E department. Currently he is professor in the department of electronics and communication, IARE, Hyderabad. His research interests include signal processing, signal estimation, probability random variables, analog and digital communications. Dr.Krishnamohan is a fellow of IETE, IE, and member of ISTE, involved in Curriculum revision for B.TECH and M.TECH courses from academic year 200506.involved in Curriculum revision for B.TECH and M.TECH courses from academic year 2005-06.Chairman of Board of Studies of E.C.E department Bottom of For

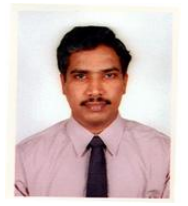

Dr.Mallikarjun S Holi was Born on 15th August 1970 at Gulbarga, completed BE in Instrumentation Technology from PDA College of Engineering (Gulbarga University) in 1993. He obtained M.Tech. and Ph.D. degree from Indian Institute Technology, Madras in 1997 \& 2004 respectively. He has 17 years of teaching experience and 8 years of Research experience in the area of Instrumentation Technology \& Biomedical Engineering. His research interests are: Biomechanics, Rehabilitation Engineering, Biomedical Signal and Image Processing, Biomedical Instrumentation \& Process Control. He has published 52 research papers in 
National \& International journals and conferences. Currently he is Professor and Chairman, Department of Electronics and Instrumentation Engineering, University B.D.T College of Engineering, Davangere, Karnataka guiding 5 students for Ph.D. Programme. He is carrying out three Sponsored Research Projects funded by AICTE and Visveswaraiah Technological University. Dr.Mallikarjun S Holi is a life member of Indian Society for Technical Education, Indian Society of Biomechanics, Bio medical Engineering Society of India, Ultrasound Society of India, Indian Association of Biomedical Scientists. Dr.Holi has received many awards, among prominent are:

- Institute Merit Award for the Best Academic record in M.Tech (Biomedical Engineering) program for the academic year 1995-1996, from I.I.T, Madras

- Received Travel Grant Award from IEEEEngineering in Medicine and Biology Society for Best Research Paper in the $28^{\text {th }}$ Annual Northeast Bioengineering Conference, Drexel University, Philadelphia, USA, 2002.

- Received Travel Grant Award from International Bone and Mineral Society \& Japanese Society for Bone and Mineral Research for Best Research Paper in the First Joint Meeting of IBMS-JSBMR, Osaka, Japan, 2003. 